\title{
Molecular surveillance of the Pfmdr1 N86Y allele in P. falciparum isolates from Brazzaville, Republic of Congo
}

\section{Louis Regis Dossou-Yovo}

Universite Marien Ngouabi

Francine Ntoumi ( $\nabla$ fntoumi@fcrm-congo.com )

Fondation Congolaise pour la Recherche Médicale https://orcid.org/0000-0003-4748-050X

Felix Koukouikila-Koussounda

Universite Marien Ngouabi

Jeannhey Christevy Vouvoungui

Fondation Congolaise pour la Recherche Medicale

Ayodele Adedoja

Fondation Congolaise pour la recherche medicale

David Nderu

Eberhard Karls Universitat Tubingen

Thirumalaisamy $P$ Velavan

Eberhard Karls Universitat Tubingen

Arsene Lenga

Universite Marien Ngouabi

\section{Research}

Keywords: P. falciparum, Pfmdr1, antimalarial drug resistance, lumefantrine, Brazzaville, Republic of Congo

Posted Date: November 28th, 2019

DOI: https://doi.org/10.21203/rs.2.17894/v1

License: (c) (1) This work is licensed under a Creative Commons Attribution 4.0 International License. Read Full License

Version of Record: A version of this preprint was published at Malaria Journal on May 8th, 2020. See the published version at https://doi.org/10.1186/s12936-020-03246-0. 


\section{Abstract}

Background: Regular surveillance of artemisinin-based combination therapy tolerance or resistance molecular makers is vital for effective malaria treatment, control and eradication programmes. P. falciparum multiple drug resistance-1 gene (Pfmdr1) N86Y mutation is associated with reduced susceptibility to lumefantrine. This study assessed the prevalence of Pfmdr1 N86Y in Brazzaville, Republic of Congo.

Methods: A total 1001 of P. falciparum-infected blood samples obtained from asymptomatic malaria pregnant women having a normal child delivery at the Madibou Integrated Health Center were analysed.Pfmdr1 N86Y genotyping was conducted using PCR-Restriction fragment length polymorphism (RFLP).

Results: The wild type Pfmdr1 N86 allele was predominant ( $>68 \%$ ) in this study whereas a few isolates carrying the either the mutant allele (Pfmdr1 86Y) alone or both alleles (mixed genotype). The dominance of the wildtype allele (pfmdr1 N86) indicates the plausible decline P. falciparum susceptibility to lumefantrine.

Conclusion: This study gives an update on the prevalence of Pfmdr1 N86Y alleles in Brazzaville, Republic of Congo. It also raises concern on the imminent emergence of resistance against artemether-lumefantrine in this setting. Our study underscores the importance to regular artemether-lumefantrine efficacy monitoring to inform malaria control programme of the Republic of Congo.

\section{Introduction}

Anti-malarial drug resistance is a major impediment to malaria reduction/eradication globally. Resistance against chloroquine (CQ) and its successor, sulfadoxine-pyrimethamine (SP), had devasting consequences in sub-Saharan Africa in 1990s and 2000, particularly among children below 5 years[1]. Currently, artemisinin-based combined therapy (ACT) is the first-line treatment for $P$. falciparum uncomplicated malaria alongside SP for intermittent preventive treatment-SP of pregnant women (ITPp) and infants (ITPi)[2].After the introduction of ACTs, malaria mortalities and morbidities global declined drastically until 2015. Even though ACTs are still highly efficacious, there is heightened concern on the potential spread of ACT resistance from Southeast Asia to sub-Saharan Africa reminiscent of the spread of CQ- and SP resistance[3-7]. The WHO recommends routine surveillance of antimalarial drug efficacy once every two years. However, high cost of clinical trials limit efforts to monitor the emergence and spread of antimalarial drug resistance in resource limited settings. Molecular surveillance of distinct point mutation(s) in $P$. falciparum genes linked to antimalaria treatment failure offers a cost-effective tool to monitor spatiotemporal emergence and spread of resistant parasites. High prevalence of gene mutations associated with $P$. falciparum CQ $(P$. falciparum chloroquine transporter gene $P f c r t)$ and SP $(P$. falciparum dihydrofolate reductase gene; $P f d h f r$ and dihydropteroate synthase gene, $P f d h p s)$ resistance 
informed, in part, the decision to replace these antimalarial drugs with ACTs including the Republic of Congo [8-11]. P. falciparum multidrug resistance1 (Pfmdr1), also known as P-glycoprotein homologue, is a transmembrane protein of the $P$. falciparum digestive vacuole (DV)[12]. It is involved transportation substrates into digestive vacuole of $P$. falciparum including antimalaria drugs[13]. Distinct changes in the sequence and/or amplification of the copy number of $P f m d r 1$ gene alters $P$. falciparumsusceptibility to several antimalarial drugs [14]. In particular, the Pfmdr1 N86Y single nucleotide polymorphism (SNP) has been implicated in $P$. falciparum resistance to chloroquine and amodiaquine [15]. Pfmdr1 N86Y is mostly found in Africa. High prevalence of $P f m d r 186 \mathrm{~N}$ and $86 \mathrm{Y}$ alleles is currently being driven by ACT-linked $P$. falciparum opposite selection. Previous studies shown that parasites carrying Pfmdr1 N86 are less susceptible lumefantrine[16, 17], artemisinin-lumefantrine, AL, select Pfmdr $186 \mathrm{~N}$ whereas artesunateamodiaquine, ASAQ, and piperaquine select $P f m d r 186 \mathrm{Y}[16,18-20]$. Since this phenomenon indicates potential decline of malaria parasite sensitivity or increased tolerance to ACT partner drugs,Pfmdr1 N86Y genotyping has been proposed as a useful marker to guide rotation of ACTs in a given geographical area[20, 21]. The present study aimed to genotype and to determine the prevalence of Pfmdr1 N89Y in Brazzaville, Republic of Congo. Data for this study is useful for the refinement and adaption of the current malaria treatment policy with the long-term goal of reducing malaria in the Republic of Congo.

\section{Methods}

\section{Sample collection}

This study analysed a total of 101 marched blood samples (maternal peripheral, placenta, and cord blood) collected from asymptomatic malaria pregnant women having a normal child delivery at the Madibou Integrated Health Center, Brazzaville, between March 2014andApril 2015(21). Brazzaville is the capital of the Republic of Congo. It is situated along the Congo River and has about 1.8 million inhabitants (22). Malaria transmission in this area is perennial with $P$. falciparum being the predominant Plasmodium specie [22, 23].AL and ASAQ are the frontline and second-line antimalarial drugs for uncomplicated $P$. falciparum malaria in the Republic of Congo, respectively [24].

\section{Pfmdr1 genotyping}

Total genomic DNA was isolated using QIAamp DNA Mini Kit (Qiagen, Hilden, Germany) according to the manufacturer's instructions. Amplification of $P$. falciparum merozoite surface protein 2 gene (Pfmsp2) was used to determine P. falciparum multiplicity of infection (MOI) as described previously [25].Nested-PCR and restriction fragment length polymorphism (PCR-RFLP) were used to genotype Pfmd1 N86Y as described previously[26]. In brief, Pfmdr1primary and nested PCRs were conducted by adding $2 \mu \mathrm{l}$ DNA template into a PCR master mix (50 $\mu$ ) containing 1 X PCR buffer $2.8 \mathrm{mM} \mathrm{MgCl}_{2}, 200 \mu \mathrm{M}$ dNTPs, 5 pM of each primer, 1 UTaq DNA polymerase. The primers for the primary PCR were A1 (5'CGGAGTGACCAAATCTGGGA-3') and A3 (5'-GGGAATCTGGTGGTAACAGC-3') and for the secondary PCR 
were A2 (5'-TTGAAGAACAGAAATTACATGATGA-3') and A4 (5'-AAAGATGGTAACCTCAGTATCAAAGAAGAG$\left.3^{\prime}\right)$. The thermal cycler conditions were as follows: initial denaturation at $94^{\circ} \mathrm{C}$ for $2 \mathrm{~min}$, followed by 40 cycles at $94^{\circ} \mathrm{C}$ for $1 \mathrm{~min}, 45^{\circ} \mathrm{C}$ for $1 \mathrm{~min}, 72^{\circ} \mathrm{C}$ for $1 \mathrm{~min}$ and a final extension at $72^{\circ} \mathrm{C}$ for $5 \mathrm{~min}$. The secondary reaction was conducted using the product of the primary reaction as a template. DNA extracted $P$. falciparum laboratory strains (3D7 and Dd2) and PCR grade water were used as positive and negative controls, respectively.

The Pfmdr1N86Y mutation was identified by digesting Pfmdr1A2/A4secondary PCR products (10 $\mu$ l) using Apol (New England Biolabs Inc. MA, USA) restriction enzyme for 15 minutes at $50^{\circ} \mathrm{C}$ according to manufacturer's instructions. The resulting DNA fragments were separated in $2 \%$ agarose gel stained with SYBR green at 100V for 45 mins electrophoresis. Apol digests Pfmdr1PCR product when Pfmdr1N86 (wild type allele) is present. Data analysesChi-square and Fisher exact tests were applied to compare the proportions of Pfmdr1 N86Y alleles in this study. The statistical significance was set at $p$-value $<0.05$. Ethical considerations

This study was approved by the Institutional Ethics Committee of Fondation Congolaise pour la Recherche Médicale, FCRM, Brazzaville, Republic of Congo. Written informed consent was obtained from all participants before samples collection. The objectives of the study including the study procedures, sample to be taken, study benefits, potential risks and discomforts were explained. Newly opened needle and syringe were used for each subject.

\section{Results}

Table 1 shows that baseline characteristics of participants recruited in this study. The mean age of participants was $23.7 \pm 5.75$ years. Overall, $24 \%$ of the pregnant women did not take intermittent preventive treatment during pregnancy (IPTp) during pregnancy and most of the participants $(70 \%)$ had $>1$ parity.

Of the 101 marched sample analysed in this study, Pfmdr1 was successfully amplified in 59 (58.4\%), 38 (37.6\%) and 21 (20.8\%) maternal peripheral blood, placental blood and cord blood samples, respectively. Pfmsp2 genotyping showed mean $\mathrm{MOI}$ was $1.06 \pm 0.24$. High prevalence of $P f m d r 1$ wild type allele (N86) was observed among the different sample types. Pfmdr1 N86 was present in $69.5 \%$ (41/59), $57.9 \%$ $(22 / 38)$ and $85.7 \%(18 / 21)$ of the peripheral blood, placenta blood and cord blood samples, respectively. The remaining samples had the mutant allele (Pfmdr186Y) with few samples having both wild type and mutant alleles. Three peripheral blood samples, two placental blood samples and one cord blood sample had both the wild type and mutant Pfmdr1 N86Y alleles. The Pfmdr1 N86 allele prevalence was similar $(p$-value $>0.05)$ among different sample types, parity and the number of Intermittent preventive treatment during pregnancy (Table 2).

\section{Discussion}


This study set out to determine the prevalence of Pfmdr1 N86Y mutation among pregnant women having a normal child delivery at the Madibou Integrated Health Center, Brazzaville Republic of Congo. The frequency of Pfmdr1 86Y(mutated allele) in this study was lower than previously estimated in this setting in 2010 (73\%) and in 2015 (27\%) [27, 28]. Our findings are also comparable to Pfmdr1 86Y allele (23\%) global frequency and most parts of Africa (17\% -24\%) except Central Africa, where higher resistant allele frequency (44\%) has been observed [29]. In Southeast Asia, however, the frequency of Pfmdr 186Y is much lower than observed in this study whereas it has almost reached fixation in Papua New Guinea[29]. Pfmdr1 N86Y mutation is known to modulate $P$. falciparum susceptibility to various antimalarial drugs by regulating the influx of the drugs into the parasite's digestive vacuole. Previous studies have shown that parasite carrying this mutation are less susceptible to 4-aminoquinolines, namely chloroquine, amodiaquine and piperaquine, in vitro [30] and increase the risk of chloroquine or amodiaquine therapeutic failure [15]. On the other hand, the Pfmdr1 86Y mutation enhance malaria parasite susceptibility to lumefantrine, mefloquine and the active derivative of artemisinin, dihydroartemisinin [30]. The converse impact of Pfmdr1 N86Y on $P$. falciparum response to longer-acting partner drugs of ACTs implies that wide spread use of artemether-lumefantrine $(A L)$ and artesunate-amodiaquine (AS-AQ),particularly in Africa, exert opposite selection pressure on $P$. falciparum populations and allele frequency $[31,32]$. Changes in malaria treatment policies greatly influence the frequency of mutations that modulate $P$. falciparum susceptibility to antimalarial drugs including Pfmdr1 N86Y[31]. The introduction of ACTs in the early 2000s and cessation of chloroquine use in the 1990s led to drastic changes in Pfmdr1 N86Y allele frequency in various malaria-endemic settings[27, 33, 34]. For instance, the frequency of Pfmdr186Y has declined dramatically, in favour of $P f m d r 1 N 86$, in countries where AL is used as the first-line treatment for malaria. The increase in Pfmdr1 N86 allele frequency is faster when $\mathrm{AL}$ is used compared to AS-AQ usage [31].In areas where AS-AQ is the primary treatment for malaria, the decline of Pfmdr1 86Y allele frequency is slow owing to reduced susceptibility of parasites carrying this mutation to amodiaquine. Previous studies demonstrate that parasites carrying Pfmdr1 N86 tolerate higher lumefantrine levels and have short-time to reinfection or recrudescence in patients with high lumefantrine concentration following $A L$ treatment $[16,17]$. Even though there is no evidence directly linking Pfmdr1 N86 to AL treatment failure and AL is still highly efficacious, parasite tolerance to lumefantrine is a clear warning signfor plausible emergence of resistance against AL. In this context, Pfmdr1 86N can be used to track lumefantrine selective pressure in a given areas[17].Our findings show that Pfmdr1N 86 allele is approaching fixation in the Republic of Congo and could provide the genetic background needed for the emergence of resistance against lumefantrine threatening AL usefulness in this setting. However, this possibility could be averted by concurrent use of AL and ASAQ as first-line treatment for uncomplicated $P$. falciparum malaria. Such a strategy is supported by evidence showing the opposite effect of both Pfmdr1 N86Y alleles on P. falciparum susceptibility to AL and ASAQ [18].

\section{Conclusions}

This study offers an update on the frequency of Pfmdr1 N86Y alleles in Brazzaville, Republic of Congo and provides evidence supporting the concomitant deployment or rotation of AL and ASAQ as the 
primary treatment for uncomplicated $P$. falciparum malaria. This will be helpful to halt any further selection of Pfmdr1 alleles that dampen parasite susceptibility and safeguard AL efficacy.

\section{Declarations}

\section{Acknowledgements}

We thank the study participants and their parents. We thank Dr Mandingha Kosso Etoka-Beka, Dr. Nerly Gampio Gueye for her contribution to sample collection and the staff of the Madibou integrated health center. FN, VTP, FKK are members of CANTAM (EDCTP-RegNet2015-1045) and PANDORA-ID-Net (Grant Agreement RIA2016E-1609) networks.

\section{Disclaimer}

The findings and conclusions in this paper are those of the authors and do not necessarily represent the views of the funding agency.

\section{Author's contributions}

LR D-Y performed all the lab analyses, FKK supervised the lab work, JCV was responsible of statistical analysis, AA and AL participated in the interpretation of the results, DN and TPV reviewed and edited the manuscript. FN supervised the overall work. All authors approved the final manuscript. All the authors contributed in drafting the paper.

\section{Funding}

This study was funded by CANTAM (EDCTP-RegNet 2015-1045) and TOTAL E\&P Congo.

\section{Availability of data and material}

All raw data provided in this work are available upon request to the corresponding author.

\section{Ethical approval and consent to participate}


This study was approved by the Institutional Ethics Committee of Fondation Congolaise pour la Recherche Médicale, FCRM, Brazzaville, Republic of Congo. Written informed consent was obtained from all participants before samples collection.

Consent for publication

Not applicable

Competing interests

Authors declare they have no competing interests.

\section{References}

1. 1. Trape JF: The public health impact of chloroquine resistance in Africa.Am J Trop Med Hyg 2001, 64:12-17.

2. WHO: Guidelines for the treatment of malaria. 3 edition. Geneva: World Health Organisation; 2015.

3. Conrad MD, Rosenthal PJ: Antimalarial drug resistance in Africa: the calm before the storm? Lancet Infect Dis 2019.

4. Hamilton WL, Amato R, van der Pluijm RW, Jacob CG, Quang HH, Thuy-Nhien NT, Hien TT, Hongvanthong B, Chindavongsa K, Mayxay M, et al: Evolution and expansion of multidrug-resistant malaria in southeast Asia: a genomic epidemiology study.Lancet Infect Dis 2019.

5. Ariey F, Witkowski B, Amaratunga C, Beghain J, Langlois AC, Khim N, Kim S, Duru V, Bouchier C, Ma L, et al: A molecular marker of artemisinin-resistant Plasmodium falciparum malaria.Nature 2014, 505:50-55.

6. Ashley EA, Dhorda M, Fairhurst RM, Amaratunga C, Lim P, Suon S, Sreng S, Anderson JM, Mao S, Sam B, et al: Spread of artemisinin resistance in Plasmodium falciparum malaria.N Engl J Med 2014, 371:411-423.

7. Mita T, Tanabe K, Kita K: Spread and evolution of Plasmodium falciparum drug resistance.Parasitol Int 2009, 58:201-209.

8. Nsimba B, Malonga DA, Mouata AM, Louya F, Kiori J, Malanda M, Yocka D, Oko-Ossho J, EbataMongo S, Le Bras J: Efficacy of sulfadoxine/pyrimethamine in the treatment of uncomplicated Plasmodium falciparum malaria in Republic of Congo.Am J Trop Med Hyg 2004, 70:133-138.

9. Mayengue PI, Ndounga M, Davy MM, Tandou N, Ntoumi F: In vivo chloroquine resistance and prevalence of the pfcrt codon 76 mutation in Plasmodium falciparum isolates from the Republic of Congo.Acta Trop 2005, 95:219-225. 
10. Ndounga M, Tahar R, Basco LK, Casimiro PN, Malonga DA, Ntoumi F: Therapeutic efficacy of sulfadoxine-pyrimethamine and the prevalence of molecular markers of resistance in under 5-year olds in Brazzaville, Congo. Trop Med Int Health 2007, 12:1164-1171.

11. Koukouikila-Koussounda F, Bakoua D, Fesser A, Nkombo M, Vouvoungui C, Ntoumi F: High prevalence of sulphadoxine-pyrimethamine resistance-associated mutations in Plasmodium falciparum field isolates from pregnant women in Brazzaville, Republic of Congo.Infect Genet Evol 2015, 33:32-36.

12. Cowman AF, Karcz S, Galatis D, Culvenor JG: A P-glycoprotein homologue of Plasmodium falciparum is localized on the digestive vacuole.J Cell Biol 1991, 113:1033-1042.

13. Reiling SJ, Rohrbach P: Monitoring PfMDR1 transport in Plasmodium falciparum.Malar J 2015, 14:270.

14. Price RN, Uhlemann AC, Brockman A, McGready R, Ashley E, Phaipun L, Patel R, Laing K, Looareesuwan S, White NJ, et al: Mefloquine resistance in Plasmodium falciparum and increased pfmdr1 gene copy number.Lancet 2004, 364:438-447.

15. Picot S, Olliaro P, de Monbrison F, Bienvenu AL, Price RN, Ringwald P: A systematic review and metaanalysis of evidence for correlation between molecular markers of parasite resistance and treatment outcome in falciparum malaria.Malar J 2009, 8:89.

16. Otienoburu SD, Maiga-Ascofare O, Schramm B, Jullien V, Jones JJ, Zolia YM, Houze P, Ashley EA, Kiechel JR, Guerin PJ, et al: Selection of Plasmodium falciparum pfcrt and pfmdr1 polymorphisms after treatment with artesunate-amodiaquine fixed dose combination or artemether-lumefantrine in Liberia.Malar J 2016, 15:452.

17. Sisowath C, Stromberg J, Martensson A, Msellem M, Obondo C, Bjorkman A, Gil JP: In vivo selection of Plasmodium falciparum pfmdr $186 \mathrm{~N}$ coding alleles by artemether-lumefantrine (Coartem).J Infect Dis 2005, 191:1014-1017.

18. Sondo P, Derra K, Diallo Nakanabo S, Tarnagda Z, Kazienga A, Zampa O, Valea I, Sorgho H, OwusuDabo E, Ouedraogo JB, et al: Artesunate-Amodiaquine and Artemether-Lumefantrine Therapies and Selection of Pfcrt and Pfmdr1 Alleles in Nanoro, Burkina Faso.PLoS One 2016, 11:e0151565.

19. Malmberg M, Ferreira PE, Tarning J, Ursing J, Ngasala B, Bjorkman A, Martensson A, Gil JP: Plasmodium falciparum drug resistance phenotype as assessed by patient antimalarial drug levels and its association with pfmdr1 polymorphisms.J Infect Dis 2013, 207:842-847.

20. Taylor AR, Flegg JA, Holmes CC, Guerin PJ, Sibley CH, Conrad MD, Dorsey G, Rosenthal PJ: Artemether-Lumefantrine and Dihydroartemisinin-Piperaquine Exert Inverse Selective Pressure on Plasmodium falciparum Drug Sensitivity-Associated Haplotypes in Uganda.Open Forum Infect Dis 2017, 4:ofw229.

21. Gil JP, Krishna S: pfmdr1 (Plasmodium falciparum multidrug drug resistance gene 1): a pivotal factor in malaria resistance to artemisinin combination therapies.Expert Rev Anti Infect Ther 2017, 15:527543. 
22. Trape JF, Peelman P, Morault-Peelman B: Criteria for diagnosing clinical malaria among a semiimmune population exposed to intense and perennial transmission. Trans $R$ Soc Trop Med Hyg 1985, 79:435-442.

23. Trape JF, Zoulani A: Malaria and urbanization in central Africa: the example of Brazzaville. Part Il: Results of entomological surveys and epidemiological analysis. Trans R Soc Trop Med Hyg 1987, 81 Suppl 2:10-18.

24. MOH: Ministère de la Santé et de la Population: Politique nationale de lutte contre le paludisme. Brazzaville, Republic of Congo2006.

25. Gueye NSG, Ntoumi F, Vouvoungui C, Kobawila SC, M NK, Mouanga AM, Deibert J, KoukouikilaKoussounda F: Plasmodium falciparum merozoite protein-1 genetic diversity and multiplicity of infection in isolates from Congolese children consulting in a pediatric hospital in Brazzaville.Acta Trop 2018, 183:78-83.

26. Mungthin M, Khositnithikul R, Sitthichot N, Suwandittakul N, Wattanaveeradej V, Ward SA, NaBangchang $\mathrm{K}$ : Association between the pfmdr1 gene and in vitro artemether and lumefantrine sensitivity in Thai isolates of Plasmodium falciparum.Am J Trop Med Hyg 2010, 83:1005-1009.

27. Koukouikila-Koussounda F, Jeyaraj S, Nguetse CN, Nkonganyi CN, Kokou KC, Etoka-Beka MK, Ntoumi F, Velavan TP: Molecular surveillance of Plasmodium falciparum drug resistance in the Republic of Congo: four and nine years after the introduction of artemisinin-based combination therapy.Malar $J$ 2017, 16:155.

28. ACT Partner Drug Molecular Surveyor [https://www.wwarn.org/tracking-resistance/act-partner-drugmolecular-surveyor]

29. P. falciparum Community Project [www.malariagen.net/projects/p-falciparum-community-project]

30. Veiga MI, Dhingra SK, Henrich PP, Straimer J, Gnadig N, Uhlemann AC, Martin RE, Lehane AM, Fidock DA: Globally prevalent PfMDR1 mutations modulate Plasmodium falciparum susceptibility to artemisinin-based combination therapies.Nat Commun 2016, 7:11553.

31. Okell LC, Reiter LM, Ebbe LS, Baraka V, Bisanzio D, Watson OJ, Bennett A, Verity R, Gething P, Roper C, Alifrangis M: Emerging implications of policies on malaria treatment: genetic changes in the Pfmdr-1 gene affecting susceptibility to artemether-lumefantrine and artesunate-amodiaquine in Africa.BMJ Glob Health 2018, 3:e000999.

32. Venkatesan M, Gadalla NB, Stepniewska K, Dahal P, Nsanzabana C, Moriera C, Price RN, Martensson A, Rosenthal PJ, Dorsey G, et al: Polymorphisms in Plasmodium falciparum chloroquine resistance transporter and multidrug resistance 1 genes: parasite risk factors that affect treatment outcomes for $P$. falciparum malaria after artemether-lumefantrine and artesunate-amodiaquine.Am J Trop Med Hyg 2014, 91:833-843.

33. Hayward R, Saliba KJ, Kirk K: pfmdr1 mutations associated with chloroquine resistance incur a fitness cost in Plasmodium falciparum.Mol Microbiol 2005, 55:1285-1295.

34. Moyeh MN, Njimoh DL, Evehe MS, Ali IM, Nji AM, Nkafu DN, Masumbe PN, Barbara AT, Ndikum VN, Mbacham WF: Effects of Drug Policy Changes on Evolution of Molecular Markers of Plasmodium 
falciparum Resistance to Chloroquine, Amodiaquine, and Sulphadoxine-Pyrimethamine in the South West Region of Cameroon.Malar Res Treat 2018, 2018:7071383.

\section{Tables}

Table 1: Baseline characteristics of participants.

\begin{tabular}{lll}
\hline & No. of participants $(\mathrm{n}=101)$ & Percentage (\%) \\
\hline Age group (years) & & \\
\hline $15-30$ & 89 & 88.1 \\
\hline$>30$ & 12 & 11.9 \\
\hline Dose of IPTp & & \\
\hline 0 & 24 & 23.8 \\
\hline 1 & 28 & 27.7 \\
\hline$\geq 2$ & 49 & 48.5 \\
\hline Parity & & \\
\hline Primipare & 30 & 29.7 \\
\hline Secondipare & 33 & 32.7 \\
\hline Multiparous & 38 & 37.6 \\
\hline
\end{tabular}

IPTp: Intermittent preventive treatment in pregnancy.

Table 2: Distribution of the Pfmdr1N86 allele among peripheral blood, placenta blood and cord blood samples from the Republic of Congo.

\begin{tabular}{llll}
\hline \multicolumn{5}{l}{ Peripheral blood n (\%) } & Placental blood n (\%) & Cord blood n (\%) \\
\hline Dose IPTp & & & \\
\hline 0 & $8 / 14(57.1)$ & $6 / 9(66.7)$ & $5 / 6(83.3)$ \\
\hline 1 & $13 / 18(72.2)$ & $4 / 13(33.3)$ & $8 / 10(80)$ \\
\hline$>2$ & $20 / 27(74.1)$ & $12 / 16(71.4)$ & $5 / 5(100)$ \\
\hline$p$-value & 0.512 & 0.251 & 0.569 \\
\hline Parity & & & $7 / 9(77.8)$ \\
\hline Primipare & $13 / 18(72.2)$ & $4 / 10(40)$ & $6 / 6(100)$ \\
\hline Secondipare & $12 / 18(66.7)$ & $10 / 10(100)$ & $5 / 6(83.3)$ \\
\hline Multiparous & $16 / 23(69.6)$ & $8 / 18(44.4)$ & 0.475 \\
\hline$p$-value & 0.936 & 0.007 &
\end{tabular}

IPTp: Intermittent preventive treatment in pregnancy. 
Page 11/11 